\title{
The incidence of vasoplegia in adult patients with right-sided congenital heart defects undergoing cardiac surgery and the correlation with serum vasopressin concentrations
}

\author{
Erica D. Wittwer, MD, PhD, ${ }^{a}$ James J. Lynch, MD, ${ }^{a}$ William C. Oliver, Jr, MD, ${ }^{a}$ Joseph A. Dearani, MD, ${ }^{b}$ \\ Harold M. Burkhart, MD, and William J. Mauermann, MD ${ }^{\mathrm{a}}$ \\ Background: In adults with right-sided congenital heart disease, vasoplegia during and after cardiopulmonary \\ bypass appears to be a frequent complication. The incidence of vasoplegia in the general adult and pediatric car- \\ diac surgical population has been investigated, but the incidence in adult patients with right-sided congenital \\ heart disease is unknown. Perioperative vasopressin levels during cardiac surgery have been studied in other car- \\ diac surgical patients, but are not known in adults with right-sided congenital heart disease. The purpose of this \\ study was to investigate the incidence of vasoplegia in adult patients undergoing right-sided cardiac surgical pro- \\ cedures requiring cardiopulmonary bypass and to determine the vasopressin response to cardiac surgery in this \\ population.
}

\begin{abstract}
Methods: Twenty patients were enrolled and demographic, hemodynamic, cardiopulmonary bypass, and use of vasoactive medication data were collected. In addition, perioperative serum vasopressin levels were measured. Sixty adult patients undergoing left-sided cardiac surgery served as controls.

Results: The incidence of vasoplegia in the control patients was $10 \%$ and the incidence in the adult patients with right-sided congenital heart disease was $20 \%$. Vasopressin levels were low at baseline $(0.5 \pm 0.5 \mathrm{pg} / \mathrm{mL})$, increased slightly after induction of anesthesia $(0.6 \pm 0.6 \mathrm{pg} / \mathrm{mL})$, increased after initiation of cardiopulmonary bypass $(99.7 \pm 168.2 \mathrm{pg} / \mathrm{mL})$, and decreased after surgery $(31.3 \pm 43.6 \mathrm{pg} / \mathrm{mL})$.

Conclusions: This study showed that the incidence of vasoplegia (20\%) in patients with right-sided congenital heart disease undergoing cardiac surgery was double that of a population of patients undergoing aortic valve surgery $(10 \%)$. Serum vasopressin concentration was not associated with vasoplegia in this population of congenital cardiac surgical patients. (J Thorac Cardiovasc Surg 2014;148:625-30)
\end{abstract}

Vasoplegia has been well described after surgery requiring cardiopulmonary bypass (CPB) as well as during off-pump coronary artery bypass grafting and is defined by hypotension in the setting of low systemic vascular resistance with normal or increased cardiac output. ${ }^{1}$ The incidence of vasoplegia in adults undergoing cardiac surgery is between $5 \%$ and $25 \% .^{2}$ Vasoplegia has been associated with increased intensive care unit length of stay and hospital length of stay. ${ }^{1,3,4}$ The type of surgery may influence the development of vasoplegia because valve procedures have

\footnotetext{
From the Departments of Anesthesiology ${ }^{\mathrm{a}}$ and Surgery, ${ }^{\mathrm{b}}$ Mayo Clinic College of Medicine, Rochester, Minn.

This project was supported by grant number UL1 TR000135 from the National Center for Advancing Translational Sciences. Its contents are solely the responsibility of the authors and do not necessarily represent the official views of the National Institutes of Health.

Disclosures: Dr Oliver reports grant support from GTC and Nonin Medical Inc. All other authors have nothing to disclose with regard to commercial support.

Received for publication June 3, 2013; revisions received Sept 19, 2013; accepted for publication Oct 11, 2013; available ahead of print Nov 25, 2013.

Address for reprints: William J. Mauermann, MD, Department of Anesthesiology, Mayo Clinic, 200 First St SW, Rochester, MN 55905 (E-mail: mauermann. william@mayo.edu).

$0022-5223 / \$ 36.00$

Copyright (c) 2014 by The American Association for Thoracic Surgery

http://dx.doi.org/10.1016/j.jtcvs.2013.10.037
}

been shown to have a higher occurrence of vasoplegia compared with coronary artery bypass grafting. ${ }^{2}$

In addition, patients with right-sided congenital heart disease are also at risk for hepatic congestion and subsequent hepatic dysfunction, which may result in a vasodilated hyperdynamic state. Vasoplegia has been investigated in patients undergoing a variety of cardiac surgeries, but patients with right-sided congenital heart disease including tetralogy of Fallot have not been studied previously. Anecdotally, in our practice there appeared to be a higher incidence of vasoplegia in patients with right-sided congenital cardiac valvular disease undergoing repair using CPB as compared with other cardiac surgical procedures.

Low levels of vasopressin may contribute to vasodilatory shock after cardiac surgery. ${ }^{5}$ In patients without vasoplegia, vasopressin levels have been shown to increase on bypass, decrease after CPB, and then increase again, and postoperative hypertension is associated with increased levels of vasopressin. ${ }^{6}$ In patients undergoing cardiac surgery, an average amount of vasopressor or inotropic support is not known and different practices use a variety of medications for hemodynamic support. The purpose of the current article is to describe the incidence of vasoplegia in adults with right-sided congenital valve disease undergoing 


\section{Abbreviations and Acronyms \\ ACE-I $=$ angiotensin-converting enzyme inhibitor \\ $\mathrm{ARB}=$ angiotensin II receptor blocker \\ $\mathrm{CPB}=$ cardiopulmonary bypass \\ $\mathrm{CVP}=$ central venous pressure}

surgical repair as compared with patients with left-sided valve disease. We hypothesized that the incidence of vasoplegia in this population would be higher than in the general cardiac surgical population and possibly would be associated with an underlying vasopressin deficiency.

\section{METHODS}

After institutional review board approval and verbal consent, patients with right-sided congenital heart disease undergoing surgical procedures requiring CPB were enrolled and prospectively evaluated for the occurrence of vasoplegia and levels of vasopressin were measured. These levels were compared with hemodynamics during CPB for potential correlation with hypotension during bypass. Assuming that the true incidence of vasoplegia in the general cardiac surgical population is between $10 \%$ and $15 \%$, 20 patients were enrolled. Also after institutional review board approval, a chart review was performed on 60 patients undergoing left-sided cardiac surgical procedures requiring CPB to determine the incidence of vasoplegia in this cardiac surgical population and to establish the dose of vasopressor associated with vasoplegia on CPB.

\section{Inclusion/Exclusion Criteria}

Patients older than the age of 18 years presenting for cardiac surgery at the Mayo Clinic (Rochester, Minn) were considered for inclusion. Patients were eligible for inclusion if they had a diagnosis of tetralogy of Fallot, pulmonary atresia, Ebstein's anomaly, or other congenital cardiac disease with primary involvement of the right-sided cardiac valves. Patients undergoing surgeries not requiring $\mathrm{CPB}$ were excluded, as were patients with other causes of vasodilatory shock including sepsis. Subjects were recruited in the Cardiovascular Surgery Clinic in cooperation with the cardiovascular surgeons and the Anesthesia Clinical Research Unit.

To evaluate the range of vasopressor use in a similar adult cardiac surgical population, 60 consecutive patients who had undergone left-sided, noncongenital cardiac surgical procedures were reviewed retrospectively to serve as a control group. As the patients with right-sided congenital heart disease underwent pulmonary valve replacement/repairs with some having right ventricular outflow tract repairs performed, adult patients undergoing left-sided cardiac surgical procedures including aortic valve replacement/repair and/or ascending aorta replacement/repairs were included. Patients undergoing coronary artery bypass grafting were excluded because differences in the incidence of vasoplegia have been shown in patients undergoing valvular surgery versus coronary artery bypass grafting. ${ }^{2}$ Patients were included if the surgical procedure also included mitral valve replacement or repair, left atrial appendage ligation, or pulmonary vein isolation. Patients requiring hypothermic circulatory arrest, descending aortic repair or replacement, or right-sided cardiac procedures were excluded from this control group.

\section{Cardiopulmonary Bypass Management}

The cannulation sites for CPB were at the discretion of the cardiac surgeon and dependent on the surgery. The aorta was cannulated with a patient sizeappropriate cannula. Venous cannulation was obtained with either a single 2 -stage cannula in the right atrium or separate cannulae in the superior and inferior vena cavae. Anticoagulation was initiated with $400 \mathrm{U} / \mathrm{kg}$ of heparin and maintained according to an activated clotting time (iSTAT1; Abbott Labs,
Abbott Park, Ill) of more than 500 seconds. Perfusion during CPB used nonpulsatile flow with Sarns 9000 (Terumo Cardiovascular Systems, Ann Arbor, Mich) CPB equipment and a Terumo membrane oxygenator. This equipment uses a hydrophilic interface between the blood and tubing. Smaller circuitry and oxygenators were used for patients with a body surface less than $2.0 \mathrm{~m}^{2}$. The CPB circuit was primed with 1000 to $1400 \mathrm{~mL}$ of Plasmalyte (Baxter Healthcare, Deerfield, Ill), mannitol (50 g), albumin (25 g), and heparin $(10,000 \mathrm{U})$. CPB flows were maintained at $2.4 \mathrm{~L} / \mathrm{min} / \mathrm{m}^{2}$. Mean arterial pressure was maintained at 60 to $80 \mathrm{~mm} \mathrm{Hg}$ during CPB. For patients undergoing aortic cross-clamping, hyperkalemic cold blood cardioplegia was given every 20 to 30 minutes for myocardial protection. Cardioplegia consisted of blood mixed with crystalloid components in a ratio of 4 parts blood to 1 part crystalloid. The crystalloid component consisted of lactated Ringers solution with $50 \mathrm{mEq} / \mathrm{L}$ of sodium bicarbonate and either $100 \mathrm{mEq} / \mathrm{L}$ (for induction of cardiac arrest) or $50 \mathrm{mEq} / \mathrm{L}$ (for maintenance of cardiac arrest) of potassium. Warm, potassium-free restorative cardioplegia was not administered before cross-clamp removal.

\section{Anesthetic Management}

Care of these cardiac surgical patients including invasive hemodynamic monitoring and vasoactive medication administration was driven largely by institutional protocols. General anesthesia was induced with propofol or ketamine. Anesthesia was maintained with isoflurane, fentanyl, midazolam, and vecuronium for neuromuscular blockade. Glucose was measured after CPB was commenced and at 30- to 60-minute intervals thereafter. Per institutional protocol, glucose values greater than $180 \mathrm{mg} / \mathrm{dL}$ were treated with an insulin infusion. Transfusion administration was guided by a departmental algorithm with defined transfusion triggers. Administration of inotropic agents or vasopressors were recorded in the anesthesia record as per usual practice.

\section{Laboratory Draws}

Study patients underwent 5 blood draws that were performed via preexisting intravascular access. The baseline blood samples for study levels were drawn either after placement of an intravenous or arterial line before or immediately after anesthesia induction. The second blood draw was obtained 30 minutes after anesthesia induction or 30 minutes after the baseline blood sample. The third blood draw was performed 30 minutes after the institution of $\mathrm{CPB}$; the fourth blood draw was performed 30 minutes after weaning from bypass; and the fifth blood draw was performed 24 hours after the end of surgery.

Demographic data were obtained including age, sex, height, weight, and sternotomy number. Liver congestion was assessed by recording liver function tests if performed, the presence of ascites, and baseline central venous pressure (CVP).

\section{Outcomes}

The primary outcome was the incidence of vasoplegia during CPB (assessed from the need for vasopressors) and the correlation between plasma levels of vasopressin and vasoplegia during CPB. Our practice is not to use pulmonary artery catheters in this population of patients with right-sided congenital cardiac disease, however, per our routine, patients on CPB were maintained with a cardiac index of $2.4 \mathrm{~L} / \mathrm{min} / \mathrm{m}^{2}$. This provided a constant cardiac index and vasoplegia could be ascertained from the use of vasopressors to treat patients while on CPB. Phenylephrine was the first vasopressor administered for low mean arterial pressure on CPB, followed by vasopressin if needed. To determine a control vasopressor dose, 60 patients undergoing left-sided cardiac surgical procedures as described earlier were assessed retrospectively for total vasopressor use on CPB and CPB duration. A vasoplegia incidence of $10 \%$ to $15 \%$ could be expected from previous literature descriptions. The phenylephrine dose in milligrams was added to the vasopressin dose in units and this value was divided by the duration of CPB in minutes (vasopressor/CPB duration ratio). To determine those with vasoplegia, a cut-off ratio was calculated from the 
mean ratio plus one standard deviation. Additional outcomes measured were mortality during hospitalization, cerebrovascular accident, renal failure requiring dialysis of any duration, myocardial infarction, respiratory failure after extubation requiring noninvasive ventilation or re-intubation, and postoperative infections.

\section{Data Analysis}

Values of plasma vasopressin levels were expressed as the mean \pm standard deviation. Values were compared between the vasoplegic group and the nonvasoplegic group using the Student $t$ test. A $P$ value less than .05 was considered significant.

\section{RESULTS}

Twenty patients with right-sided congenital heart disease completed the study. Baseline demographic information is shown in Table 1. There were 11 men and 9 women, and their mean age was $31.2 \pm 10.9$ years of age. Sixteen of the 20 patients underwent repairs related to tetralogy of Fallot. Only 1 patient was undergoing a primary sternotomy, with the remainder undergoing repeat sternotomies (mean sternotomy number, $2.7 \pm 1.3$; range, 1-4). Vasopressin levels for the 20 patients are shown in Figure 1.

Sixty patients undergoing elective left-sided aortic valvular procedures were evaluated to assess the use of vasopressors and the incidence of vasoplegia in this population and to determine a vasoplegic versus nonvasoplegic vasopressor/CPB duration ratio. This population included 37 men $(62 \%)$, with an average age of $68.5 \pm 13.8$ years. Other demographics included mean sternotomy number $(1.2 \pm 0.6)$ and mean $\mathrm{CPB}$ duration $(78.0 \pm 32 \mathrm{~min})$. Figure 2, $A$, shows the vasopressor/CPB duration ratios for these 60 patients. The mean ratio plus one standard deviation $(0.07+0.15)$ was 0.22 . Thus, a vasopressor/CPB ratio of 0.22 or less was used to indicate no vasoplegia, and a ratio greater than 0.22 was used to indicate the presence of vasoplegia. Six of the 60 patients had vasopressor/CPB ratios greater than 0.22 , yielding a vasoplegia incidence of $10 \%$ in this population of cardiac surgical patients.

The vasopressor/CPB duration ratio was calculated in the 20 congenital patients (Figure 2,B). By using the previously determined ratio of greater than 0.22 as an indicator of vasoplegia, 4 of the 20 patients $(20 \%)$ were deemed vasoplegic during $\mathrm{CPB}$. When comparing the vasoplegic congenital patients with the nonvasoplegic congenital patients there were no difference between the patient demographics or plasma vasopressin levels at the baseline, second, fourth, and fifth blood draws (Tables 1 and 2). There was a statistically significant difference between the vasoplegic and nonvasoplegic patients during the third blood draw, which occurred 30 minutes after initiating $\mathrm{CPB}$, with the vasoplegic patients having higher levels of vasopressin owing to exogenous vasopressin administration (Table 2). Differences in ascites, liver function tests, and CVP were examined in patients with and without vasoplegia. One patient in each group was reported to have ascites and the baseline CVP values were not different between the groups. Liver function tests were measured in less than half of the patients and there was no difference in values between the 2 groups (data not shown).

There were significant demographic differences between the patients with congenital heart disease and the patients undergoing left-sided cardiac surgical procedures. On average, the patients with congenital heart disease were younger, had more sternotomies performed, and had shorter durations on CPB. Patients undergoing left-sided cardiac surgical procedures had a higher incidence of antihypertensive use, including a higher incidence of angiotensinconverting enzyme inhibitor (ACE-I) and angiotensin II receptor blocker (ARB) use compared with the patients with congenital heart disease (Table 1). The difference between ACE-I and ARB use was not statistically different between congenital patients with and without vasoplegia. In the patients undergoing left-sided procedures the use of an ACE-I or ARB was not statistically different between the vasoplegic and nonvasoplegic patients, however, the use of any form of antihypertensive therapy was higher in vasoplegic patients $(P<.05)$. Hemoglobin levels, temperature, glucose values, and incidence of hyperglycemia treatment on cardiopulmonary bypass as well as measures of clinical outcomes for the different groups can be seen in Table 3. In

TABLE 1. Demographics of vasoplegic and nonvasoplegic patients with right-sided congenital heart disease compared with vasoplegic and nonvasoplegic noncongenital patients who underwent left-sided cardiac surgical procedures

\begin{tabular}{lcccc}
\hline & $\begin{array}{c}\text { Congenital vasoplegic } \\
\text { patients }(\mathbf{N}=\mathbf{4})\end{array}$ & $\begin{array}{c}\text { Congenital nonvasoplegic } \\
\text { patients }(\mathbf{N}=\mathbf{1 6})\end{array}$ & $\begin{array}{c}\text { Left-sided vasoplegic } \\
\text { patients }(\mathbf{N}=\mathbf{6})\end{array}$ & $\begin{array}{c}\text { Left-sided nonvasoplegic } \\
\text { patients }(\mathbf{N}=\mathbf{5 4})\end{array}$ \\
\hline Male patients, N (\%) & $3(75 \%)$ & $8(50 \%)$ & $5(83 \%)$ & $32(59 \%)$ \\
Age, mean + SD (y) & $25 \pm 7$ & $33 \pm 11$ & $62 \pm 19$ & $69 \pm 13$ \\
Height, mean + SD (cm) & $170 \pm 6$ & $168 \pm 10$ & $176 \pm 8$ & $170 \pm 11$ \\
Weight, mean \pm SD (kg) & $73 \pm 17$ & $77 \pm 19$ & $113 \pm 34$ & $87 \pm 25$ \\
Sternotomy number, mean \pm SD & $2.0 \pm 0$ & $2.8 \pm 1.4$ & $68 \pm 28$ & $1 \pm 0$ \\
CPB duration, mean \pm SD, min & $60 \pm 34$ & $61 \pm 23$ & $6(100 \%)$ & $79 \pm 33$ \\
Antihypertensive use, N (\%) & $1(25 \%)$ & $5(31 \%)$ & $4(67 \%)$ & $39(72 \%)$ \\
ACE-I or ARB use, N (\%) & 0 & $1(6 \%)$ & $25(46 \%)$ \\
\hline
\end{tabular}

$N$, Number of patients; $S D$, standard deviation; $C P B$, cardiopulmonary bypass; $A C E-I$, angiotensin-converting enzyme inhibitor; $A R B$, angiotensin II receptor blocker. 


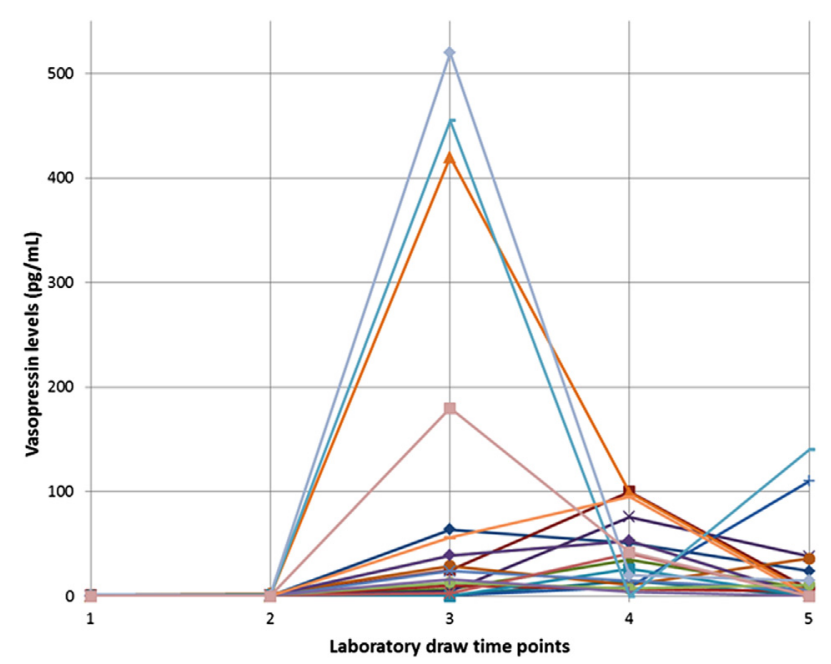

FIGURE 1. Laboratory blood draw time points and vasopessin levels.

the first 24 hours after surgery, there was no difference in the use of inotropic or vasopressor agents between the vasoplegic and nonvasoplegic right-sided congenital heart disease patients.

\section{DISCUSSION}

The key findings of this study are that the incidence of vasoplegia during $\mathrm{CPB}$ in patients undergoing right-sided congenital valve surgery is 2 times that of patients undergoing left-sided cardiac surgery. This increased incidence of vasoplegia does not appear to be caused by a deficiency of endogenous vasopressin.

Multiple factors may influence the development of vasoplegia. Blood pressure and vascular control is multifactorial, with humoral factors working together and in opposition to ultimately result in vascular tone. Vasopressin causes vasoconstriction by acting at the vasopressin 1 receptor after
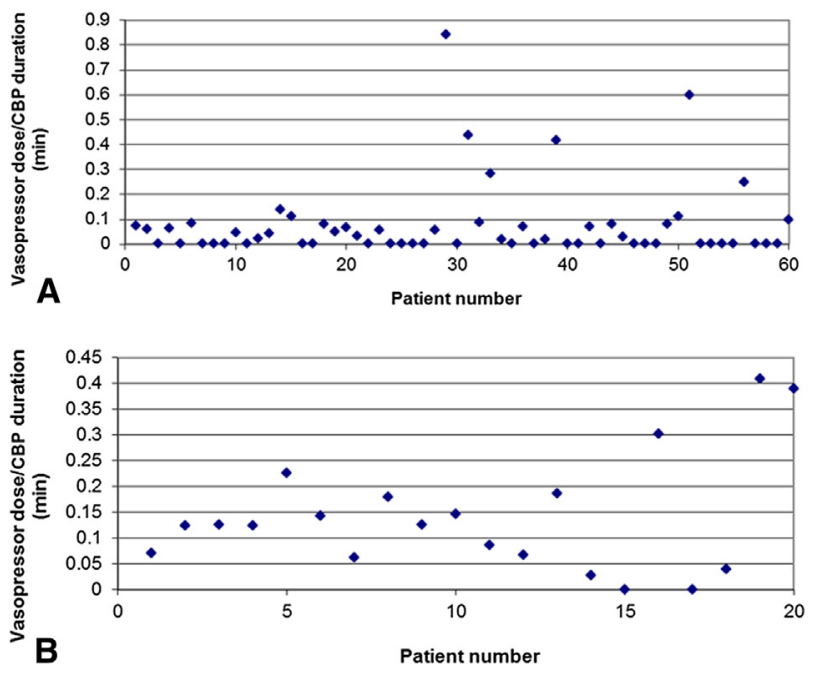

FIGURE 2. A and B, Total vasopressor dose per cardiopulmonary bypass duration ratio. being synthesized in the hypothalamus and released from the pituitary gland. ${ }^{7,8}$ In a study comparing 33 patients with vasodilatory shock with 10 patients without shock after cardiac surgery, vasopressin levels were lower in patients with vasodilatory shock on the first postoperative day, whereas by the third postoperative day the vasopressin levels were higher in the group with vasodilatory shock than in the control group. ${ }^{5}$

Normal values of vasopressor use on CPB were not available in the literature even though previous investigators had selected their own cut-off values for inotrope and vasopressor use to qualify for vasoplegia, specifically $4 \mathrm{U} / \mathrm{h}$ or more of vasopressin. ${ }^{9}$ The mean vasopressor use/CPB duration plus one standard deviation (0.22) was used as the cut-off value for vasoplegia in this study based on the analysis of 60 patients undergoing left-sided cardiac surgery. This value fit graphically with the data as well as provided a $10 \%$ incidence of vasoplegia, which is consistent with previous literature (Figure 2). The 6 patients who were in the vasoplegic category received the equivalent of $4 \mathrm{U} / \mathrm{h}$ or more of vasopressin or very high doses of phenylephrine on CPB. This is consistent with the definition of vasoplegia used in the study mentioned previously. ${ }^{9}$ When this methodology was applied to the right-sided congenital heart disease patients, a $20 \%$ incidence of vasoplegia was calculated. This supports our hypothesis that patients undergoing right-sided congenital heart surgery have a higher incidence of vasoplegia: twice that of our left-sided aortic valve replacement or repair population.

The source of an increase in vasoplegia in patients with right-sided congenital heart disease is not known. Several factors may influence the high incidence of vasoplegia in this population including age or a relative overproduction of vasodilating mediators or underproduction of vasoconstricting mediators. Although we noted an increase in vasoplegia in these patients, it is interesting to note that reoperation has been reported to be protective against vasoplegia. ${ }^{9}$ Even with an increase in this potentially protective factor, an increased percentage of these patients were vasoplegic. It is also of interest that the congenital heart disease patients had a higher incidence of vasoplegia despite a lower use of ACE-Is or ARBs, medications that have been associated with vasoplegia. ${ }^{9}$

Our second hypothesis was that patients with right-sided congenital heart disease may suffer from liver congestion, causing a vasodilatory state, or a deficiency in vasopressin could be resulting in hypotension, however, there was no statistically significant difference in CVP, presence of ascites, or liver function tests between the patients with vasoplegia versus those without vasoplegia. In addition, aside from the third blood sample, there was no difference in vasopressin levels before, during, or after surgery between those with vasoplegia and those without. The third blood sample showed a significant difference between vasopressin levels 
TABLE 2. Mean vasopressin levels for all right-sided congenital heart disease patients and levels for the nonvasoplegic versus vasoplegic patients

\begin{tabular}{|c|c|c|c|c|}
\hline & $\begin{array}{c}\begin{array}{c}\text { All congenital } \\
\text { patients }(N=20)\end{array} \\
\end{array}$ & $\begin{array}{c}\text { Vasoplegic } \\
\text { patients }(N=4) \\
\end{array}$ & $\begin{array}{c}\begin{array}{c}\text { Nonvasoplegia } \\
\text { patients }(N=16) \\
\end{array} \\
\end{array}$ & $\begin{array}{c}\text { Vasoplegic versus } \\
\text { nonvasoplegic patients } \\
\end{array}$ \\
\hline Vasopressin levels (pg/mL) & Mean \pm SD & Mean \pm SD & Mean \pm SD & $P$ value \\
\hline Baseline blood draw & $0.5 \pm 0.5$ & $0.5 \pm 0.6$ & $0.5 \pm 0.5$ & 1 \\
\hline Second blood draw & $0.6 \pm 0.6$ & $0.3 \pm 0.3$ & $0.6 \pm 0.6$ & 4 \\
\hline Third blood draw & $99.7 \pm 168.2$ & $302.8 \pm 220.9$ & $45.6 \pm 105.0$ & .003 \\
\hline Fourth blood draw & $40.6 \pm 32.9$ & $52.3 \pm 38.6$ & $38.4 \pm 32.7$ & .5 \\
\hline Fifth blood draw & $31.3 \pm 43.6$ & $77.5 \pm 88.4$ & $22.9 \pm 35.6$ & .1 \\
\hline
\end{tabular}

The baseline blood draw was drawn after entering the operating room or after induction of anesthesia; the second blood draw was 30 minutes after induction of anesthesia; the third blood draw was 30 minutes after going on cardiopulmonary bypass; the fourth blood draw was 30 minutes after separation from cardiopulmonary bypass; and the fifth blood draw was 24 hours after the end of the procedure. $S D$, Standard deviation.

for patients with vasoplegia and this would be expected because vasoplegic patients had all received vasopressin boluses at the time of this blood draw and most of those without vasoplegia had not received boluses of vasopressin. Overall, in this population of patients with right-sided congenital heart disease, vasopressin levels initially were low, increasing slightly after the onset of anesthesia, increasing dramatically after the onset of CPB, and subsequently decreasing after surgery. This is consistent with the vasopressin response in other populations of adult cardiac surgical patients. ${ }^{10}$ This also is consistent with the vasopressin response in children undergoing cardiac surgery requiring $\mathrm{CPB}$ : levels have been shown to increase while on bypass, stay high for 12 hours, begin to decrease at 24 hours, and return to baseline at 48 hours. Hypotension was not associated with a vasopressin deficiency in these children. ${ }^{11}$

Study limitations included difficulty measuring cardiac output in this population of patients without pulmonary artery catheters, which limited the assessment of vasoplegia to the CPB period. Although there was not a significant difference in inotropic and vasopressor use in the first 24 hours after surgery, it was not possible to measure cardiac output or systemic vascular resistance, making a definitive diagnosis of vasoplegia not possible. Further work examining cardiac output with noninvasive methods after surgery is needed. Another limitation was being unable to differentiate between endogenous and exogenous vasopressin. The vasoplegic patients had higher levels of vasopressin on $\mathrm{CPB}$, but this likely was owing to the administration of vasopressin. Therefore, the levels of endogenous vasopressin in these patients are unknown and it is unknown if the levels were different compared with the nonvasoplegic patients. A third limitation was the difference in age between the congenital heart disease patients and the patients undergoing left-sided cardiac surgery. Although age alone does not appear to be a strong risk factor for the development of vasoplegia, this is a confounder. The mean age of the congenital patient population was 31.2 years, and it is difficult to find a population of patients without congenital heart disease undergoing cardiac surgery at this age.

In conclusion, this study of 20 patients with right-sided congenital heart disease showed an incidence of vasoplegia of $20 \%$, twice the incidence found in our left-sided aortic valve population. Plasma vasopressin levels were not different in vasoplegic versus nonvasoplegic patients, and

TABLE 3. Demographic variables on cardiopulmonary bypass and outcome data for the congenital patients with and without vasoplegia and the noncongenital patients undergoing left-sided surgical procedures with and without vasoplegia

\begin{tabular}{lcccc}
\hline & $\begin{array}{c}\text { Congenital vasoplegic } \\
\text { patients }\end{array}$ & $\begin{array}{c}\text { Congenital nonvasoplegic } \\
\text { patients }\end{array}$ & $\begin{array}{c}\text { Left-sided vasoplegic } \\
\text { patients }\end{array}$ & $\begin{array}{c}\text { Left-sided nonvasoplegic } \\
\text { patients }\end{array}$ \\
\hline CPB variables & & & & \\
Hemoglobin (g/dL), mean \pm SD & $11.5 \pm 4.3$ & $9.8 \pm 1.4$ & $10.3 \pm 0.9$ & $9.2 \pm 1.4$ \\
Temperature (Celsius), mean \pm SD & $36.2 \pm 0.8$ & $36.3 \pm 0.6$ & $35.8 \pm 1.1$ & $35.0 \pm 1.6$ \\
Glucose range (mg/dL), mean \pm SD & $97-227$ & $93-300$ & $116-174$ & $79-323$ \\
Insulin infusion, N (\%) & $128 \pm 35$ & $128 \pm 44$ & $142 \pm 18$ & $128 \pm 26$ \\
Postoperative outcomes & $1(25 \%)$ & $4(25 \%)$ & & $11(20 \%)$ \\
Mortality, N (\%) & 0 & 0 & 0 & 0 \\
CVA, N (\%) & 0 & 0 & $1(17 \%)$ & 0 \\
Renal failure, N (\%) & 0 & 0 & 0 & $1(2 \%)$ \\
MI, N (\%) & 0 & 0 & 0 & 0 \\
Respiratory failure, N (\%) & 0 & $1(6 \%)$ & 0 & $1(2 \%)$ \\
Infection, N $(\%)$ & 0 & $1(6 \%)$ & 0 & $1(2 \%)$ \\
\hline
\end{tabular}

$S D$, Standard deviation; $N$, number of patients; $C V A$, cerebral vascular accident; $M I$, myocardial infarction. 
the vasopressin response to cardiac surgery was similar to other cardiac surgical populations with levels beginning low, increasing slightly after the onset of anesthesia, increasing dramatically after the onset of CPB, and subsequently decreasing after surgery. This study provides insight into the incidence of vasoplegia in this special cardiac surgical population of patients with right-sided congenital heart disease and suggests areas for further clinical investigations in these patients.

\section{References}

1. Sun X, Boyce SW, Herr DL, Hill PC, Zhang L, Corso PJ, et al. Is vasoplegic syndrome more prevalent with open-heart procedures compared with isolated onpump CABG surgery? Cardiovasc Revasc Med. 2011;12:203-9.

2. Fischer GW, Levin MA. Vasoplegia during cardiac surgery: current concepts and management. Semin Thorac Cardiovasc Surg. 2010;22:140-4.

3. Mekontso-Dessap A, Houel R, Soustelle C, Kirsch M, Thebert D, Loisance DY. Risk factors for post-cardiopulmonary bypass vasoplegia in patients with preserved left ventricular function. Ann Thorac Surg. 2001;71:1428-32.
4. Carrel T, Englberger L, Mohacsi P, Neidhart P, Schmidli J. Low systemic vascular resistance after cardiopulmonary bypass: incidence, etiology, and clinical importance. J Card Surg. 2000;15:347-53.

5. Jochberger S, Velik-Salchner C, Mayr VD, Luckner G, Wenzel V, Falkensammer G, et al. The vasopressin and copeptin response in patients with vasodilatory shock after cardiac surgery: a prospective, controlled study. Intensive Care Med. 2009;35:489-97

6. Feddersen K, Aurell M, Delin K, Haggendal J, Aren C, Radegran K. Effects of cardiopulmonary bypass and prostacyclin on plasma catecholamines, angiotensin II and arginine-vasopressin. Acta Anaesthesiol Scand. 1985;29: 224-30.

7. Holmes CL, Landry DW, Granton JT. Science review: vasopressin and the cardiovascular system part 1-receptor physiology. Crit Care. 2003;7:427-34.

8. Holmes CL, Landry DW, Granton JT. Science review: vasopressin and the cardiovascular system part 2-clinical physiology. Crit Care. 2004;8:15-23.

9. Levin MA, Lin HM, Castillo JG, Adams DH, Reich DL, Fischer GW. Early oncardiopulmonary bypass hypotension and other factors associated with vasoplegic syndrome. Circulation. 2009;120:1664-71.

10. Yamane Y, Yamadori Y, Umeda Y, Shiota T. Plasma ADH levels during heart surgery. Jpn Circ J. 1979;43:263-75.

11. Morrison WE, Simone S, Conway D, Tumulty J, Johnson C, Cardarelli M. Levels of vasopressin in children undergoing cardiopulmonary bypass. Cardiol Young. 2008;18:135-40. 